\title{
Top Quark Pair Cross Section Measurements at CMS
}

\author{
Jan KIESELER ${ }^{* \dagger}$ \\ Deutsches Elektronen-Synchrotron (DESY), Notkestr. 85, 22607 Hamburg, Germany \\ E-mail: jan.kieseleredesy.de
}

\begin{abstract}
Measurements of inclusive and differential top-quark pair production cross sections at a center of mass energy of 8 and $7 \mathrm{TeV}$ are presented. The total cross section is measured in the lepton+jets and the dileptonic decay modes. Differential cross sections are obtained as a function of various kinematic observables, including the transverse momentum and rapidity of the (anti)top quark, kinematics of the top-antitop system, and jet multiplicity in the event. The precise results are used to extract the strong coupling constant from inclusive top-pair production cross sections. All measurements use LHC data collected by the CMS experiment in 2011 and 2012.
\end{abstract}

XXI International Workshop on Deep-Inelastic Scattering and Related Subject -DIS2013, 22-26 April 2013

Marseilles, France

\footnotetext{
*Speaker.

${ }^{\dagger}$ On behalf of the CMS Collaboration
} 


\section{Introduction}

A precise measurement of $\mathrm{t} \overline{\mathrm{t}}$ cross sections in proton-proton (pp) collisions at the Large Hadron Collider (LHC) offers a benchmark for the production mechanism of processes dominated by gluon-gluon fusion. Moreover, it can be used to validate calculations in quantum chromodynamics or to provide constraints on standard model parameters and parton distribution functions. An accurate understanding of the t⿱t production and decay is also crucial for searches for new physics processes that show a similar event topology.

The inclusive and differential cross section measurements presented in this note use pp collision data collected with the CMS detector [1] in 2011 and 2012 at a center of mass energy of 7 or $8 \mathrm{TeV}$, respectively.

\section{Inclusive Cross Section Measurements}

With full NNLO predictions up to a precision of $6 \%$ available [2], precise measurements of the inclusive cross section are important tests for the underlying theory. These are performed in distinct decay modes of the t⿱亠t pair and are described here with a focus on recent results at $\sqrt{s}=8 \mathrm{TeV}$. In all measurements, the event selection is based on the event topology of the particular decay mode categorized by the decay of the $W$ bosons from $t \bar{t} \rightarrow W^{+} W^{-} b \bar{b}$.

\subsection{Dileptonic Decay Modes}

In the dilepton decay modes, where both $W$-bosons decay to a lepton and a neutrino, final states incorporating $e^{ \pm} e^{\mp}, e^{ \pm} \mu^{\mp}$ or $\mu^{ \pm} \mu^{\mp}$ are studied [3]. Consequently, events are selected by identifying at least two oppositely charged isolated leptons with high transverse momentum $\left(p_{T}\right)$ and at least two high- $p_{T}$ jets of which at least one is identified as $b$-jet. Events with a low dilepton mass are removed in order to supress heavy-flavour resonance decays. Contributions from $Z^{0}$ production are reduced by excluding the $Z^{0}$ dilepton mass window and requiring a high amount of $E_{T}$ in the $e^{+} e^{-}$and $\mu^{+} \mu^{-}$channels. The dominant background from Drell-Yan events and from non- $W / Z^{0}$ leptons are estimated from data, single-top-quark production and diboson background events are taken from simulation.

The $\mathrm{t} \overline{\mathrm{t}}$ production cross section at $\sqrt{s}=8 \mathrm{TeV}$ is measured to be $\sigma_{t \bar{t}}=227 \pm 3$ (stat.) \pm 11 (syst.) \pm 10 (lumi) pb, for a top-quark mass of $172.5 \mathrm{GeV}$. The dominant systematic uncertainties are jet-energy calibration and lepton efficiencies.

\subsection{Semileptonic Decay Modes}

The semileptonic $t \bar{t}$ decay modes are characterized by one $W$ boson decaying leptonically and one decaying hadronically. Events are selected that yield exactly one isolated, identified lepton and at least four high- $p_{T}$ jets of which one is identified as a b-jet [4]. The shape of the QCD multijet background is estimated from a control region with non-isolated leptons. The latter, the $\bar{t} \bar{t}$ signal shape and the remaining background (from simulation) is used in a binned likelihood fit of the lepton-b-jet invariant mass to extract the $t \bar{t}$ cross section.

For a top-quark mass of $172.5 \mathrm{GeV}$ and $\sqrt{s}=8 \mathrm{TeV}$, the t⿱t cross section is measured to be $\sigma_{t \bar{t}}=229 \pm 9$ (stat.) $\pm_{26}^{29}$ (syst.) \pm 10 (lumi) pb. Systematic uncertainties arise mainly from theory 
uncertainties, b-tagging and jet-energy calibration. A comparison to theory predictions for both measurements presented is shown in Figure 1 as well as a summary of measured inclusive $t \bar{t}$ cross sections at $\sqrt{s}=7 \mathrm{TeV}$. All measurements are in good agreement with predictions.
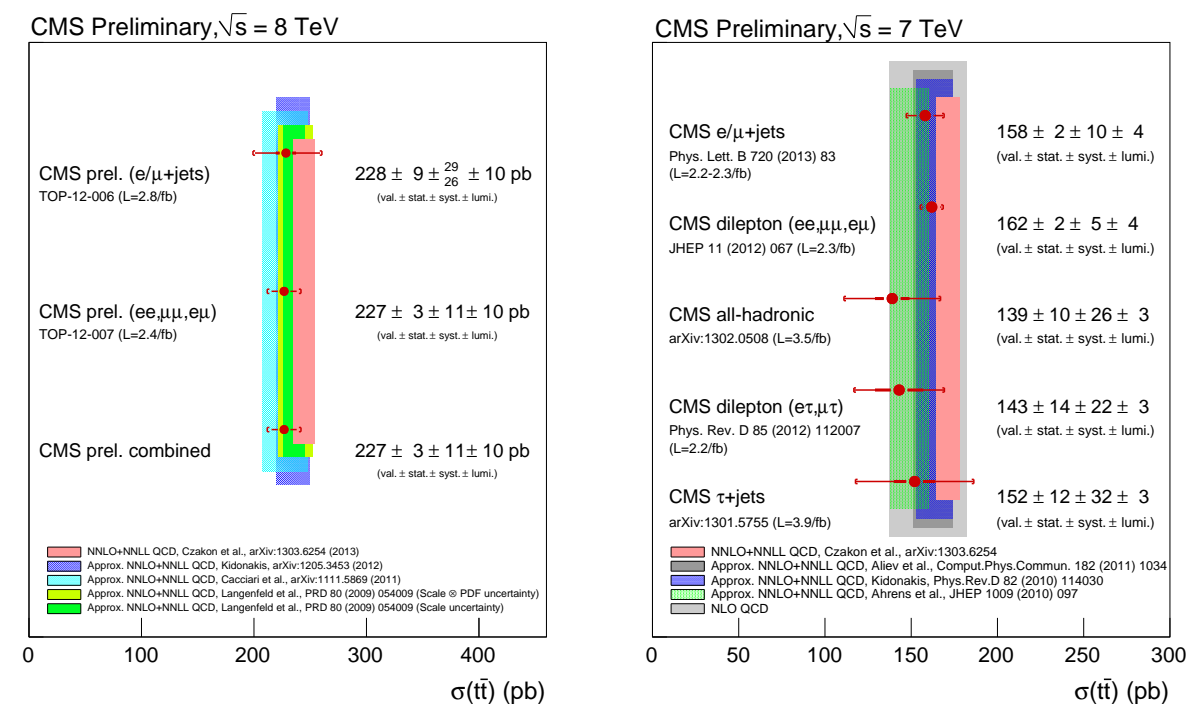

Figure 1: Summary of cross section measurements performed with the CMS detector compared to NNLO and approx. NNLO predictions, shown by shaded bands, at $\sqrt{s}=8 \mathrm{TeV}$ (left) and $\sqrt{s}=7 \mathrm{TeV}$ (right).

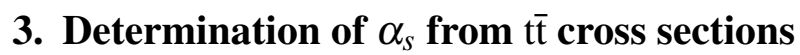

The strong coupling constant $\alpha_{s}$ is the only free parameter in the QCD Lagrangian besides the quark masses. The dependence of $\alpha_{s}(Q)$ on the energy scale of a process $Q$ is given by the renormalization group equation and typically evolved to $Q=m_{Z}$. Only very few measurements allow $\alpha_{s}$ to be tested at high scales, e.g. $Q \geq m_{t}$.

The production of $t \bar{t}$ pairs at high scales and its precisely measured cross section [5] can be combined with predictions at NNLO [2] to extract $\alpha_{s}$ [6]. The method is taking advantage of an expected significantly enhanced sensitivity of $\sigma_{t \bar{t}}$ to $\alpha_{s}$ due to correlations with the gluon parton density function (PDF). For different PDFs, a joined likelihood fit is performed using the predicted cross section as a function of $\alpha_{s}\left(m_{Z}\right)$ and the measured $t \bar{t}$ cross section. For the first time, by constraining the pole-top mass to the latest average from direct measurements and using the PDF set NNPDF2.3, a value of $\alpha_{s}\left(m_{Z}\right)=0.1151 \pm_{0.0032}^{0.0033}$ is extracted, in agreement with the world average.

\section{Differential Cross Section Measurements}

The high $t \bar{t}$ production rate at the LHC allows for measurements of the $t \bar{t}$ production cross section as a function of various kinematic observables. These differential cross sections are important tests for QCD predictions, enhance the sensitivity to new physics, and can be used for PDF constraints. The measurements at $\sqrt{s}=8 \mathrm{TeV}$ presented here are performed in the dileptonic [7] and the semileptonic decay modes [8] and follow a similar event selection as the inclusive crosssection measurements. A kinematic reconstruction of the top-pair system is performed and cross 
sections are extracted using a regularized unfolding technique. The results are normalized to the in-situ measured cross section to reduce normalization uncertainties. In general, all distributions show good agreement between data and theory predictions (MADGRAPH interfaced with Pythia, MC@NLO interfaced with Herwig, POWHEG interfaced with Pythia) as shown in Figure 2. The normalized cross section as a function of top $p_{T}$ seems to favor approx. NNLO predictions, similar to measurements at $\sqrt{s}=7 \mathrm{TeV}[9]$.
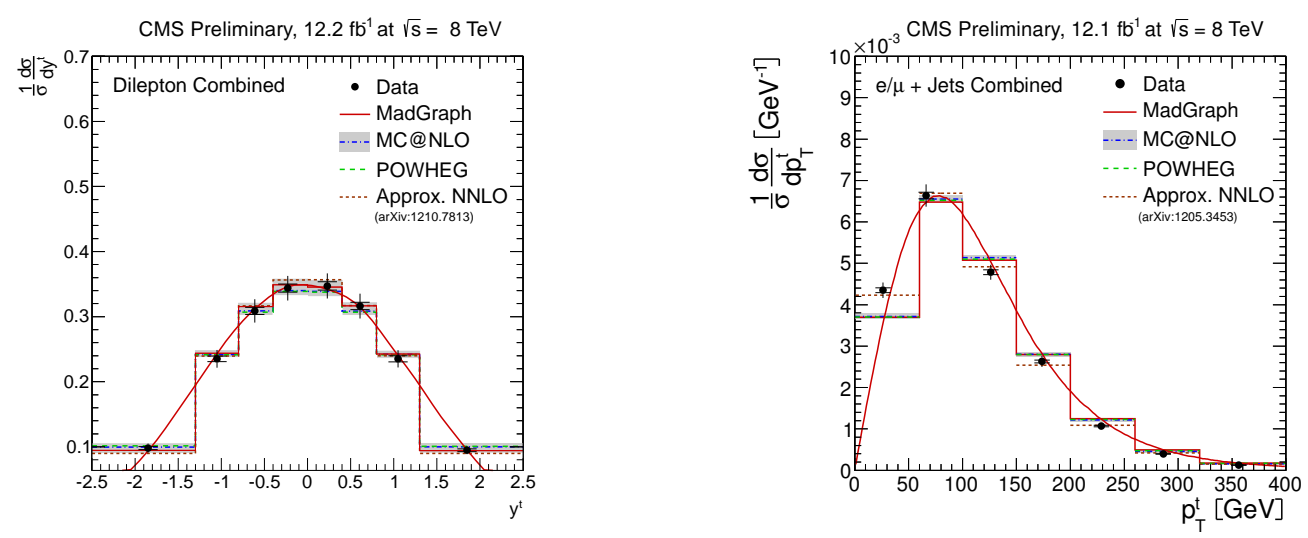

Figure 2: Normalized differential $\bar{t} \bar{t}$ production cross section as a function of top rapidity $\left(y_{t}\right)$ measured in the dileptonic decay mode (left) and as a function of top transverse momentum $\left(p_{T}\right)$ measured in the semileptonic decay mode (right) compared to different predictions (MADGRAPH interfaced with Pythia, MC@NLO interfaced with Herwig, POWHEG interfaced with Pythia, and approx NNLO). Data is presented as filled symbols. Inner (outer) error bars indicate statistical (combined statistical and systematic) uncertainties.

\subsection{Jet Multiplicities in Top Pair Events}

A high fraction of events at the LHC yields additional hard jets from initial and final state radiation (ISR, FSR). The understanding of ISR and FSR is crucial not only for high energetic top or Higgs production but for all processes involving high jet multiplicities. A measurement of tt production with additional jets is performed in in the semileptonic decay mode at $\sqrt{s}=7 \mathrm{TeV}$ [10] and the dileptonic decay mode at $\sqrt{s}=8 \mathrm{TeV}$ [11]. Figure 3 shows an increasing difference between several predictions (MadGraph interfaced with Pythia, MC@NLO interfaced with Herwig, POWHEG interfaced with Pythia) with increasing jet multiplicity. For high multiplicities MC@NLO interfaced with Herwig seems to underestimate the data in both decay modes.

\section{Summary}

A variety of cross section measurements has been performed using pp collision data collected with the CMS experiment at a center of mass energy of 7 and $8 \mathrm{TeV}$. Inclusive top pair cross sections have been measured in nine decay channels in total and up to a high precision, in good agreement with predictions. These precise results were used to extract the strong coupling constant from the $t \bar{t}$ cross section. Differential cross sections were measured in the dileptonic and semileptonic decay modes and provide a basis for precision test of the Standard Model and searches for new physics. 

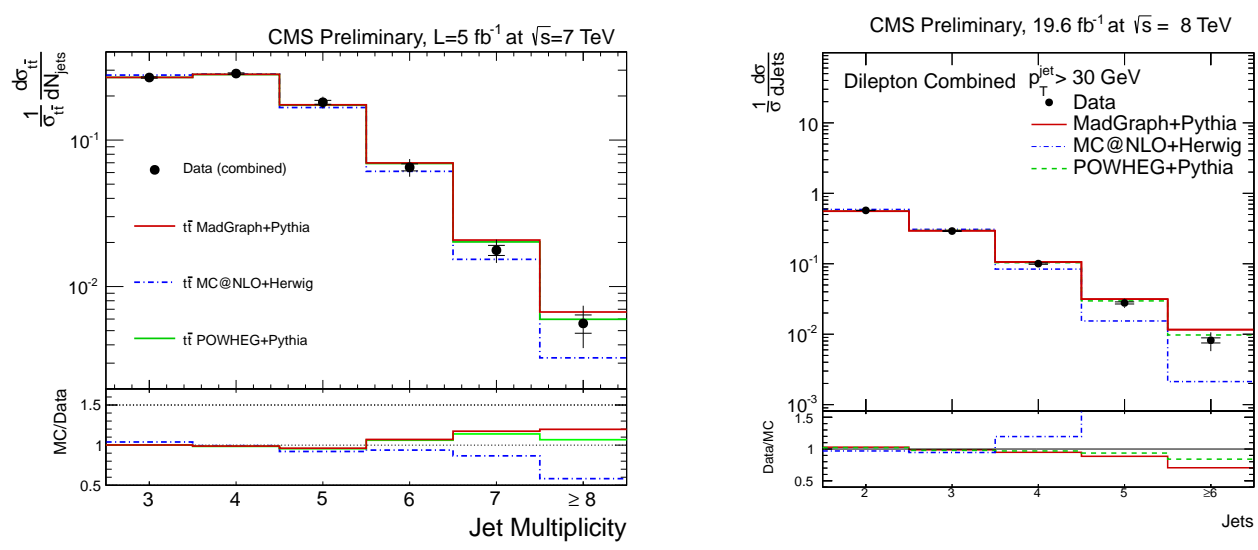

Figure 3: Normalized differential $t \bar{t}$ production cross section as a function of jet multiplicity measured in the dileptonic decay mode (left) [11] and the semileptonic decay mode (right) [10] compared to different predictions (MADGRAPH interfaced with Pythia, MC@NLO interfaced with Herwig, POWHEG interfaced with Pythia). Data is presented as filled symbols. The inner (outer) error bars indicate statistical (combined statistical and systematic) uncertainties.

\section{References}

[1] The CMS Collaboration, The CMS experiment at the CERN LHC, JINST 03 (2008) S08004

[2] Michal Czakon, Paul Fiedler, Alexander Mitov The total top quark pair production cross-section at hadron colliders through $O\left(\alpha_{s}^{4}\right), \mathrm{CERN}-\mathrm{PH}-\mathrm{TH} / 2013-056$ [arXiv:1303.6254 [hep-ph]]

[3] The CMS Collaboration, Top pair cross section in dileptons, CMS-PAS-TOP-12-007

[4] The CMS Collaboration, Top pair cross section in e/mu+jets at $8 \mathrm{TeV}$, CMS-PAS-TOP-12-006

[5] The CMS Collaboration, Measurement of the ttbar production cross section in the dilepton channel in pp collisions at sqrt(s)=7 TeV, JHEP 11 (2012) 067 [arXiv:1208.2671 [hep-ex]]

[6] The CMS Collaboration, Determination of the top-quark pole mass and strong coupling constant from the ttbar production cross section in pp collisions at $\operatorname{sqrt}(s)=7 \mathrm{TeV}, \mathrm{CERN}-\mathrm{PH}-\mathrm{EP}-2013-121$ [arXiv:1307.1907 [hep-ex]]

[7] The CMS Collaboration, Measurement of the differential ttbar cross section in the dilepton channel at $8 \mathrm{TeV}$, CMS-PAS-TOP-12-028

[8] The CMS Collaboration, Measurement of differential top-quark pair production cross sections in the lepton+jets channel in pp collisions at $8 \mathrm{TeV}$, CMS-PAS-TOP-12-027

[9] The CMS Collaboration, Measurement of differential top-quark pair production cross sections in pp collisions at sqrt(s) = 7 TeV, Eur. Phys. J. C73 (2013) 2339 [arXiv:1211.2220 [hep-ex]]

[10] The CMS Collaboration, Measurement of jet multiplicity in top pair events, CMS-PAS-TOP-12018

[11] The CMS Collaboration, Measurement of the Jet Multiplicity in dileptonic Top Quark Pair Events at 8 $\mathrm{TeV}, \mathrm{CMS}-\mathrm{PAS}-\mathrm{TOP}-12-041$ 